$\underset{\text { clinical }}{\text { nephron }}$

Practice
Nephron 2019;142:275-283

DOI: 10.1159/000499939
Received: February 4, 2019

Accepted after revision: March 25, 2019

Published online: April 16, 2019

\title{
The Role of Uric Acid in Acute Kidney Injury
}

\author{
Abutaleb Ahsan Ejaz ${ }^{\mathrm{a}}$ Richard J. Johnson ${ }^{\mathrm{b}}$ Michiko Shimada ${ }^{\mathrm{c}}$ \\ Rajesh Mohandas $^{\text {a }}$ Kawther F. Alquadan ${ }^{a}$ Thomas M. Beaver ${ }^{d}$ \\ Vijay Lapsiae Bhagwan Dass ${ }^{a}$
}

${ }^{a}$ Division of Nephrology, Hypertension and Transplantation, University of Florida, Gainesville, FL, USA; ${ }^{b}$ Division of Renal Diseases and Hypertension, University of Colorado Anschutz Medical Campus, Aurora, CO, USA; ' ${ }^{\circ}$ ivision of Nephrology, Hirosaki University Graduate School of Medicine, Hirosaki, Japan; 'Division of Cardiovascular Surgery, University of Florida, Gainesville, FL, USA; ${ }^{e}$ Division of Nephrology, Icahn School of Medicine at Mount Sinai, New York, NY, USA

\section{Keywords}

Uric acid · Acute kidney injury · Causality

\begin{abstract}
Studies have demonstrated the presence of a strong association between serum uric acid (SUA) and acute kidney injury (AKI) consistently across several disease models. Exposure to SUA at different time periods and concentrations has reliably resulted in AKI whether assessed by conventional or novel biomarkers or by kinetic estimated glomerular filtration rate (KeGFR) engineered for non-steady dynamic states. In experimental models, moderate hyperuricemia was associated with an absence of intrarenal crystals, manifestation of tubular injury, macrophage infiltration, and increased expression of inflammatory mediators that were attenuated with uric acid lowering therapy with rasburicase, a recombinant urate oxidase. In a pilot clinical trial, treatment with rasburicase was associated with a decreased incidence of AKI and evidence for less renal structural injury. Lowering SUA also improved KeGFR and estimated glomerular filtration rate in 2 separate studies. SUA has also been linked to diabetic nephropathy, hypertension, cardiovascular disease,
\end{abstract}

chronic kidney disease, metabolic syndrome, and their mechanisms of action share many common traits. In this article, we explore the evidence for the causal role of SUA in AKI using Bradford Hill criteria as a guideline with data integration from related fields.

(c) 2019 S. Karger AG, Basel

\section{Introduction}

Scientists attribute the improvement of human life span over the millennia in part to improved protective mechanisms against oxygen radicals. In 1981, Ames proposed that one of those protective systems is serum uric acid (SUA) [1]. In humans, uric acid is a major antioxidant. Uric acid acts as an antioxidant in the extracellular environment (reacting with superoxide to make allantoin and with peroxynitrite to make triuret) and as a prooxidant in the intracellular environment. For centuries, the biological significance of uric acid is that it crystallizes in joints to cause gouty arthritis and in the urinary tract to cause kidney stones. SUA, that is, in concentrations that do not cause crystal precipitations, has been associated with hypertension, chronic kidney disease,

\section{KARGER}

(c) 2019 S. Karger AG, Basel

E-Mail karger@karger.com

www.karger.com/nef
Abutaleb Ahsan Ejaz, MD

Division of Nephrology

Hypertension and Transplantation

PO Box 100224, Gainesville, FL 32610 (USA)

E-Mail ejazaa@gmail.com 
cardiovascular diseases, stroke, diabetic nephropathy, metabolic syndrome, and acute kidney injury (AKI) [2], but until recent years was viewed primarily as a secondary feature or epiphenomenon.

SUA is an emerging risk factor for AKI. Numerous studies have linked SUA to AKI; however, its causal role in AKI remains controversial. A conclusion of causality is deemed a judgment based on a body of evidence, and the Bradford Hill criteria serve as a checklist to provide epidemiologic evidence of a causal relationship [3]. Fedak has argued that more diverse types of information obtained from advances in other scientific fields need to be considered when establishing causality beyond the traditional epidemiologic considerations proposed by Bradford Hill [4]. In this article, we systematically analyzed original researches in experimental and clinical models to explore the possibilities that SUA may have a causal role in AKI. Bradford Hill criteria were utilized as a guideline with data integration from related fields.

\section{Strength of Association of SUA and AKI}

Uric acid causes AKI in tumor lysis syndrome (TLS) by a crystal-dependent mechanism [5]. Elevated SUA has also long been associated with AKI in pediatric cardiac surgery (CS) patients where it was assumed that uric acid crystal-induced tubular obstruction was the mechanism involved. Emerging data have questioned this assumption. In a retrospective analysis of 2 randomized studies of patients with coronary artery bypass surgery (Guard during Ischemia against Necrosis; 11,590 patients and Sodium-Proton Exchange Inhibition to Prevent Coronary Events in Acute Cardiac Conditions; 5,761 patients), preand postoperative $S U A \geq 5.5 \mathrm{mg} / \mathrm{dL}$ was associated with a two- to fourfold increased risk for AKI [6]. In a prospective study of high-risk CS patients (thoracic aortic aneurysm and cardiac valves, $n=58$ ), preoperative SUA $\geq 6.1$ $\mathrm{mg} / \mathrm{dL}$ was associated with a fourfold (OR 3.98, 95\% CI 1.1-14.3) increased risk of AKI after controlling for known risk factors of AKI in CS such as baseline kidney function, left ventricular ejection fraction, type of surgery, and redo CS [7]. In a retrospective study of adult patients undergoing nontransplant, high-risk CS $(n=$ 190), SUA was demonstrated to be an independent predictor of AKI [8]. SUA $\geq 5.5 \mathrm{mg} / \mathrm{dL}$ was associated with a fourfold (OR 3.7, 95\% CI 1.8-7.3) increased risk for AKI. In a prospective observational study $(n=100)$, postoperative SUA was associated with an increased incidence of AKI and graded risk for AKI [9]. Compared to the low- est tertile, the highest tertile of SUA $(>5.77 \mathrm{mg} / \mathrm{dL})$ was associated with an eightfold (OR 7.9, 95\% CI 1.5-42.1) increased risk for postoperative AKI and a fivefold (OR 4.8, 95\% CI 1.2-19.2) increased risk for AKI during hospital stay. Table 1 lists the studies that have reported comparable results regarding the association of SUA and AKI.

SUA was demonstrated to have comparable predictive values as the conventional biomarkers serum creatinine (SCreat) and estimated glomerular filtration rate (eGFR) and novel biomarkers urine neutrophil gelatinase-associated lipocalin, urine interleukin-18, serum tumor necrosis factor-alpha, and monocyte chemoattractant protein-1 for post-cardiovascular surgery-associated AKI [9].

\section{Consistency of Association of SUA and AKI}

\section{Consistent Findings Observed by Different Persons in}

Different Places with Different Samples Strengthens the Likelihood of an Effect

Multiple studies in CS have repeatedly demonstrated a significant association between SUA and AKI regardless of methodological differences and patient characteristics (Table 1). This association was repeatedly tested and found to be consistent across different study designs in CS: in prospective $[7,9-12]$ and retrospective studies [8, 13-15], preoperative $[7-9,11,12]$ and postoperative time periods $[10,14]$, and when using conventional and novel biomarkers of AKI as endpoints [7-10, 13]. This consistent association of SUA and AKI has been demonstrated across multiple disease models. For example, in the Jerusalem Lipid Research Clinic study $(n=2,449)$, SUA was an independent predictor of AKI and CKD [16] in patients with normal kidney function followed over 24-28 years, elderly patients with chronic kidney disease [17], acute myeloid leukemia [13, 14], hospitalized patients [17-19], urological surgery $[20,21]$, radiocontrast exposure [22], and burn injury [23].

\section{Specificity of the Effect of SUA}

Causation Is Likely if there Is a Very Specific

Population at a Specific Site and Disease with No

Other Likely Explanation

That is, exposure causes one disease - a rule that is considered untenable by most researchers because it assumes that a cause has one single effect ignoring the fact that variables often represent aggregates of many characteristics. Moreover, the consideration of specificity is use- 
Table 1. Evidence for causal role of SUA in AKI

\begin{tabular}{|c|c|}
\hline Criteria & Evidence \\
\hline Strength of association & 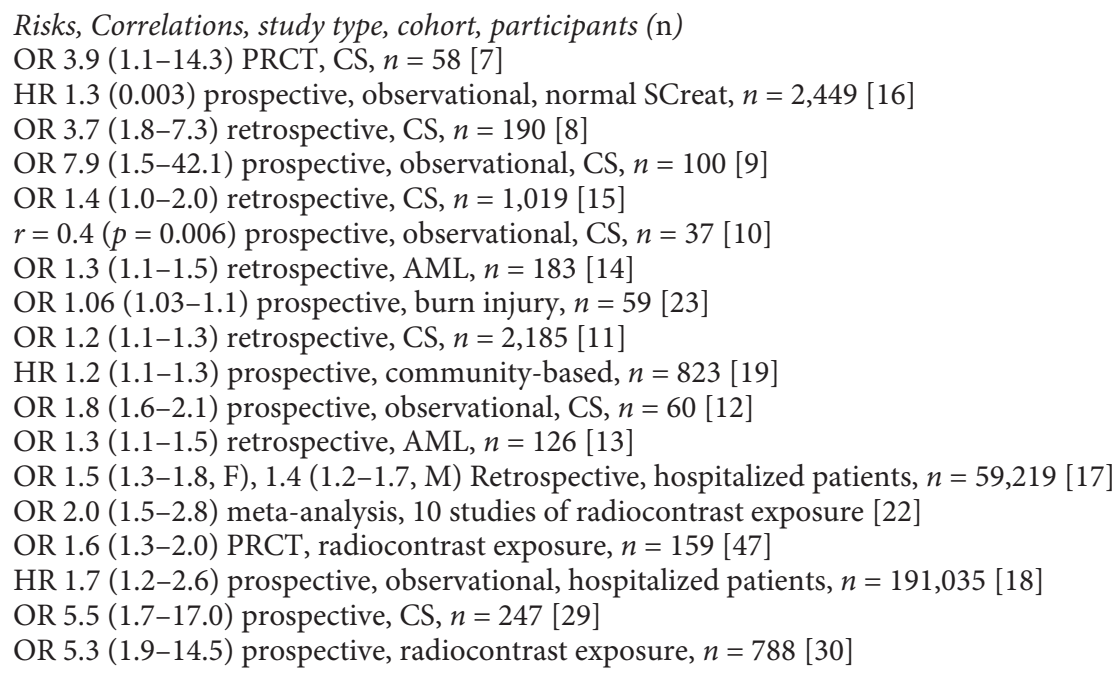 \\
\hline Consistency & $\begin{array}{l}\text { Settings } \\
\text { Cardiac surgery }[7-15,29] \\
\text { Chronic kidney disease }[16] \\
\text { Acute myeloid leukemia }[13,14] \\
\text { Radiocontrast exposure }[22,30,47,48] \\
\text { Hospitalized patients }[17-19] \\
\text { Burn [23] } \\
\text { Urology surgery/procedures }[20,21] \\
\text { Paraquat intoxication }[50]\end{array}$ \\
\hline Specificity & Criterion not met; see text for discussion \\
\hline Temporality & $\begin{array}{l}\text { SUA exposure and increased risk of AKI } \\
\text { Preoperative }[7,8,10-12,15,29,44] \\
\text { Postoperative }[9] \\
\text { Pretreatment }[13,14,45] \\
\text { Pre-contrast }[22,30,47,48] \\
\text { Dynamic }[13]\end{array}$ \\
\hline Biological gradient & $\begin{array}{l}\text { Exposure grade } \\
\text { SUA <6.1 vs. > 6.1 mg/dL: increased risk of AKI [7] } \\
\text { Deciles of SUA: incremental risk of AKI [8] } \\
\text { Tertiles of SUA: incremental risk of AKI [9] } \\
\text { Quartiles of SUA: incremental risk of AKI [12] } \\
\text { Continuous, dynamic: incremental risk of AKI [11] } \\
\text { Continuous [10] }\end{array}$ \\
\hline Plausibility and coherence & $\begin{array}{l}\text { Proposed mechanisms of SUA-induced AKI } \\
\text { Crystal-dependent mechanisms [31] } \\
\text { Crystal nephropathies [32] } \\
\text { Crystal-independent mechanisms vasoconstriction [33] } \\
\text { Decreased renal blood flow [33] } \\
\text { Decreased SNGFR [33] } \\
\text { Preglomerular arteriolopathy [33] } \\
\text { Impaired autoregulation [33] } \\
\text { VSMC proliferation [34], antiangiogenic [35] } \\
\text { Proinflammatory mediators [36] } \\
\text { Proinflammatory mediators [37] } \\
\text { Decreased bioavailability of nitric oxide [38] } \\
\text { Vascular hyper-responsiveness [38] }\end{array}$ \\
\hline
\end{tabular}


Table 1. (continued)

\begin{tabular}{ll}
\hline Criteria & Evidence \\
\hline Experiment & Study design, intervention groups, outcome \\
& Animal model, interventional study: cisplatin + hyperuricemiavs. cisplatin + hyperuricemia + rasburicase, \\
& decreased renal injury [43] \\
& Interventional study: PRCT, placebo vs. rasburicase, $n=26$, CS, decreased renal injury by biomarkers [44] \\
& Interventional study: prospective, rasburicase, $n=76$, non-Hodgkins lymphoma, reduced incidence of AKI \\
& and need for dialysis [45] \\
& Interventional study: PRCT, placebo vs. allopurinol, $n=67$, reduced incidence of AKI [46] \\
& Interventional study: PRCT, placebo vs. allopurinol, $n=159$, reduced incidence of AKI [47] \\
& Interventional study: PRCT, hydration vs. N-acetyl cysteine + hydration vs. allopurinol + hydration, $n=$ \\
& 500, reduced incidence of AKI [48] \\
& Tumor lysis syndrome [5] \\
& Crystal nephropathy [32] \\
& Mesoamerican nephropathy [49] \\
Radiocontrast nephropathy [22] & Cardiac surgery [7] \\
Analogy & Hypertension [2] \\
& Chronic kidney disease [2] \\
Cardiovascular disease [2] & Diabetes [25]
\end{tabular}

SUA, serum uric acid; AKI, acute kidney injury.

Fig. 1. Proposed mechanisms of uric acidinduced AKI. SUA in AKI. GFR, glomerular filtration rate.

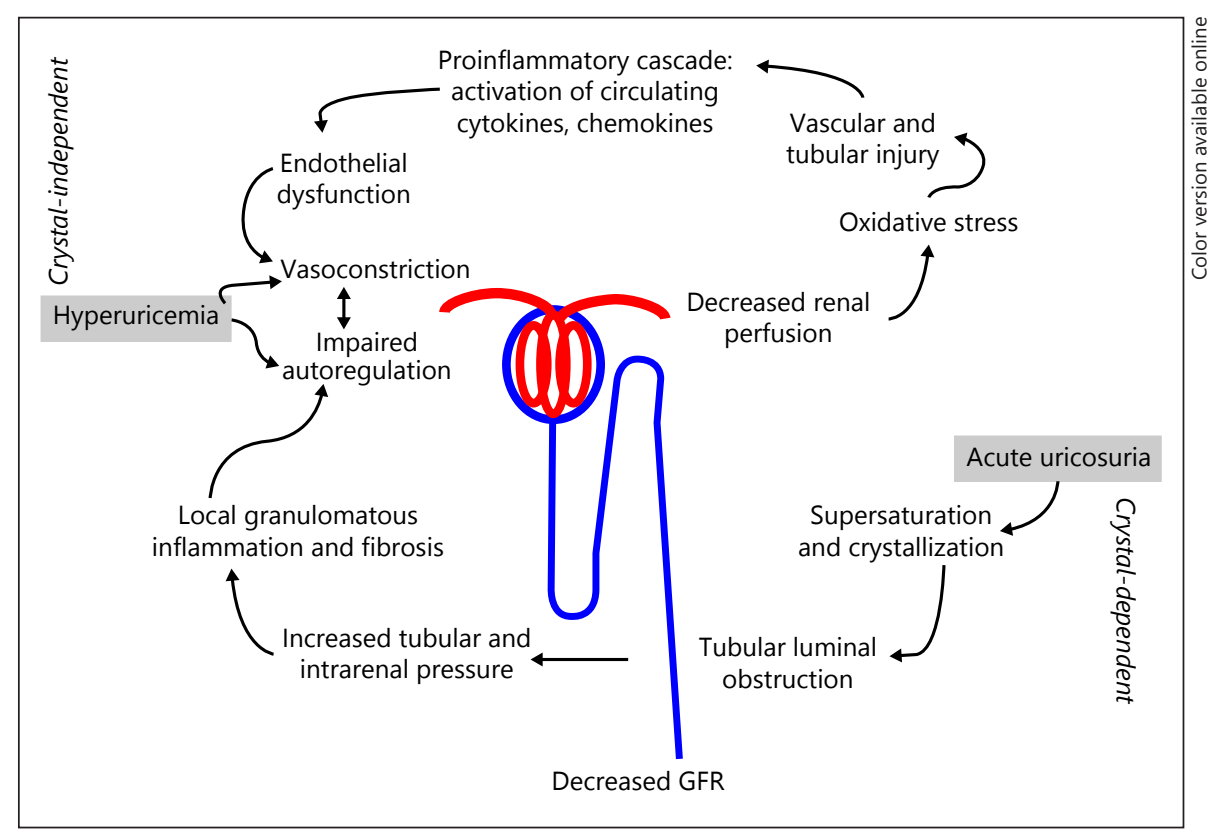

ful only when a causal system is simple and the knowledge about it is certain. Data integration framework allows for mechanisms of action to link cause and effect. SUA has been linked to hypertension, chronic kidney disease, cardiovascular diseases, stroke, diabetic nephropathy, metabolic syndrome, and AKI via mechanisms that include proinflammatory and antiangiogenic effects $[2,6]$. Many of the properties of SUA are common to different pathological states. For example, potential mechanisms of SUA-induced hypertension identified primarily from experimental studies include impaired endothelial function, stimulating endothelin-1, activating both the renal 
and intracellular renin angiotensin system, stimulating intracellular oxidative stress by activation of NADPH oxidases in the cytosol and mitochondria, stimulating smooth muscle cell proliferation and inducing inflammatory changes in the kidney that perpetuate the hypertension [24]. In the development of insulin resistance and subsequent diabetes, an elevated SUA has been reported to inhibit AMP-activated protein kinase and stimulates gluconeogenesis, blocks insulin-mediated endothelial nitric oxide release critical for insulin action, and induces oxidative stress in adipocytes leading to adiponectin synthesis [25-27]. An elevated SUA has been reported to have a contributory role in obesity and hepatic steatosis by causing intracellular and mitochondrial oxidative stress. The inhibition of aconitase in the Krebs cycle leads to citrate accumulation and stimulation of ATP citrate lyase resulting in increased fat synthesis and impaired fatty acid oxidation [28]. The proposed mechanisms for AKI associated with SUA include experimental studies that suggest an elevated serum urate can induce renal vasoconstriction, impaired autoregulation, and activation of inflammatory cascade leading to decreases in GFR [6].

\section{Temporality of SUA Exposure and AKI}

Elevated preoperative SUA predicted postoperative AKI in CS in multivariate regression models that adjusted for traditional risk factors of AKI in multiple studies [7-9, $11,12,29]$. Preoperative SUA $(>7 \mathrm{mg} / \mathrm{dL})$ in CS $(n=190)$ was associated with a 35-fold (OR 35.4, 9.7-128.7, $p<$ 0.001 ) increased risk for AKI after adjusting for type of surgery, hypertension, diabetes mellitus, coronary artery disease, previous CS, eGFR, diuretic use, $\mathrm{LVEF}<45 \%$, and cardiopulmonary bypass time [8]. In a prospective, observational study of 100 CS patients, the highest tertile of postoperative SUA was associated with an eightfold (OR 8.38, 95\% CI 2.13-33.05, $p=0.002$ ) increased risk for AKI [9]. The incidences of AKI associated with highest SUA tertile in the postoperative $0-24 \mathrm{~h}, 24-48 \mathrm{~h}$ and during hospital stay were greater compared to the lowest tertile at any given time period. When analyzed for SUA $<5.5$ $\mathrm{mg} / \mathrm{dL}$ vs. SUA $>5.5 \mathrm{mg} / \mathrm{dL}$ (the prooxidative effect manifests at SUA $>5.5 \mathrm{mg} / \mathrm{dL}$ ), the incidence of AKI was 13.1 vs. $48.7 \%(p<0.001)$.

Temporality of exposure and effect was investigated in a unique cohort of AML patients $(n=126)$ where SUA fluctuated during standard treatment [13]. SUA decreased from baseline (pre-uric acid lowering therapy and hydration) values on post-induction days 1,2,3, and
4 by $20.4,11.6,20$, and $13.7 \%$ respectively. SUA was associated with an increased risk for AKI and demonstrated a linear correlation with SCreat $(r=0.35, p<0.001)$ and an inverse correlation with kinetic eGFR (KeGFR; $r=$ $-0.33, p<0.001$ ) that persisted through several days of fluctuating SUA levels during the treatment of AML. The inverse relationship between SUA and KeGFR has also been demonstrated in CS patients [10]. SUA precedes and predicts acute changes in renal function. Since renal vasoconstriction is an initiator and propagator of AKI, these findings therefore suggest that the observed increase in KeGFR was probably associated with the reversal of renal vasoconstriction related to the reduction in SUA levels and cannot be ascribed to a simple relationship in which a reduced GFR raises SUA.

Temporality of exposure and effect was also demonstrated in several studies of radiocontrast-induced AKI. In a meta-analysis of 10 studies of radiocontrast-induced AKI, elevated SUA prior to administration of radiocontrast was associated with a twofold increased risk for AKI (pooled OR 2.03, 95\% CI 1.4-2.7) [22]. Significant heterogeneity was found in the cohort studies that included 10,427 patients who were enrolled regardless of diabetes or hypertension status and underwent coronary angiography with or without percutaneous coronary interventions. In a prospective observational study, SUA was associated with fivefold increased risk (OR 5.38, 95\% CI 1.9-14.5) for radiocontrast AKI in patients with relatively normal SCreat [30].

\section{Dose-Dependent Effect of SUA on AKI}

\section{If a Dose Response Is Seen, it Is more Likely that the} Association Is Causal

In a small $(n=58)$, prospective study of CS patients, a single preoperative uric acid level $>6.1 \mathrm{mg} / \mathrm{dL}$ (compared to SUA $<6.1 \mathrm{mg} / \mathrm{dL}$ ) conferred a nearly fourfold increased risk for AKI that was statistically significant and independent of baseline renal function, left ventricular function, type of surgery, and redo surgery [7]. In a retrospective analysis of CS patients $(n=190)$, the incidence of AKI exhibited a linear trend with increasing deciles of preoperative SUA: 1st, SUA 2.2-3.7 mg/dL, AKI 14.3\%; 2nd, SUA 3.8-4.5 mg/dL, AKI 20.5\%; 3rd, SUA 4.6-5.0 mg/dL, AKI 29.8\%; 4th, SUA 5.1-5.4 mg/dL, AKI 33.3\%; 5th, SUA 5.5-5.8 mg/dL, AKI 34\%; 6th, SUA 5.9-6.4 mg/dL, AKI 36\%; 7th, SUA 6.5-7.3 mg/dL, AKI 40\%; 8th, SUA 7.4-7.9 mg/dL, AKI 44.1\%; 9th, SUA 8.0-8.9 mg/dL, AKI 49.1\%; and 10th, SUA 9.0-13.8 mg/dL, AKI 100\% [8]. In 
the adjusted model, the risk for AKI increased with higher SUA threshold values: SUA $\geq 5.5 \mathrm{mg} / \mathrm{dL}$, OR 2.9 (1.45.9); SUA $\geq 6.0 \mathrm{mg} / \mathrm{dL}$, OR 4.1 (1.9-8.4); SUA $\geq 6.5 \mathrm{mg} /$ $\mathrm{dL}$, OR 5.6 (2.6-12.0); and SUA $\geq 7 \mathrm{mg} / \mathrm{dL}, \mathrm{OR} 35.4$ (9.7128.7). The exposure-response effect was also demonstrated in subgroups at high-risk for AKI, that is, in patients undergoing thoracic aortic aneurysm, cardiac valves or coronary artery bypass surgeries, GFR $<60 \mathrm{~mL} /$ min and left ventricular ejection fraction $<45 \%$. This relationship was nonlinear and demonstrated a U-shaped curve, characteristic of many cardiovascular risk factors (e.g., blood pressure and body mass index).

A biological gradient was also demonstrated with postoperative SUA and AKI in CS, a time period where SUA is most diluted due to intraoperative fluid administration [9]. The 1st tertile SUA $(<4.53 \mathrm{mg} / \mathrm{dL}), 2$ nd tertile SUA $(>4.53$ and $<5.77 \mathrm{mg} / \mathrm{dL})$, and 3rd tertile SUA $(>5.77 \mathrm{mg} /$ dL) was associated with $15.1,11.7$, and $54.5 \%$ incidence of AKI respectively. In a retrospective analysis of 2,185 CS patients, OR for AKI for preoperative SUA quartiles 1 ( $<$ $4.8 \mathrm{mg} / \mathrm{dL}$ for males and $<4.2 \mathrm{mg} / \mathrm{dL}$ for females), 2 ( $4.8-$ 5.5 and $4.2-4.9 \mathrm{mg} / \mathrm{dL}), 3(5.6-6.4$ and $5.0-5.8 \mathrm{mg} / \mathrm{dL}$ ), and $4(\geq 6.5$ and $\geq 5.9 \mathrm{mg} / \mathrm{dL}$ ) were 1.0 (referent), $0.93(0.7-$ $1.2), 1.26(0.9-1.6)$, and $1.61(1.2-2.0)$ respectively [11].

\section{Plausibility and Coherence}

\section{A Plausible Mechanism, Coherence between}

Epidemiological and Laboratory Findings Increases

the Likelihood of an Effect

Plausibility and coherence are discussed as one topic, as the differences between them are subtle.

The rapid development of hyperuricemia in TLS and rhabdomyolysis has been linked to AKI via intraluminal crystal precipitation (Fig. 1). The mechanism of uric acid crystal-induced AKI was demonstrated by the induction of hyperuricemia (baseline SUA $1.36 \mathrm{mg} / \mathrm{dL}$ increased to $8.13 \mathrm{mg} / \mathrm{dL}$ ) in a rat model [31]. Hyperuricemia was shown to cause dilatation of the collecting ducts with the flattening of the epithelium and intraluminal crystal precipitation, increased intraluminal hydrostatic pressures, and decreased single nephron GFR (57.6\%, micropuncture) and renal plasma flow (52.4\%, PAH clearance), thus establishing the role of uric acid in AKI via crystaldependent mechanisms. Other crystal-dependent mechanisms include the activation of the inflammasome and necroptosis, crystal adhesion, extratubulation, granuloma formation, interstitial inflammation, and tubular cell injury [32]. Additionally, local and systemic inflamma- tory responses induced by activation of inflammasomes with interleukin $1 \mathrm{~b}$ release also play significant roles in AKI.

In a breakthrough study, Sanchez-Lozada demonstrated that SUA, in concentrations that do not cause crystal precipitations, can also cause a $50 \%$ reduction in single nephron GFR and renal blood flow [33], suggesting crystal-independent mechanisms for SUA-associated AKI. Studies have since demonstrated that mild hyperuricemia stimulates proliferation and migration of vascular smooth muscle cells, and inhibits proliferation and migration, and stimulates apoptosis of proximal tubular and vascular endothelial cells $[26,34]$. SUA activates renin-angiotensin system, increases reactive oxygen radicals, inflammatory mediators $[35,36]$, and decreases the bioavailability of nitric oxide in experimental studies [34, 37, 38]. Increased SUA in animals also causes preglomerular arteriolar thickening and impairs renal autoregulation [33].

Studies in humans have also reported that lowering SUA with allopurinol can reduce plasma renin activity in adolescent hypertension [2] and improve endothelial function in a wide variety of conditions. In a meta-analysis of 11 studies that investigated the effects of allopurinol on endothelial dysfunction in hyperuricemia, treatment with allopurinol was associated with a significant increase in endothelium-dependent vasodilatation (MD $2.69 \%, 95 \%$ CI $2.4-2.8, p<0.001$; heterogeneity $\mathrm{X}^{2}=$ $319.1, I^{2}=96 \%, p<0.001$ ) [39]. The cumulative experimental and clinical data are in accordance with the proposed mechanisms of AKI that include impaired renal autoregulation related to vasoconstriction, hypoperfusion, ischemia/reperfusion injury, and the activation of the inflammatory cascade [40] and provided the stimulus to investigate the relative contribution of SUA in AKI [6, $41,42]$.

\section{Experiment: Effect of Modifying SUA on AKI}

In a model of cisplatin-induced AKI in rats, moderate hyperuricemia was associated with an absence of intrarenal crystals and manifestation of greater tubular injury and proliferation with significantly greater macrophage infiltration and increased expression of monocyte chemoattractant protein-1 [43]. Treatment with rasburicase reversed the inflammatory changes and decreased tubular injury with an improvement in renal function. These data provided the first experimental evidence that uric acid, at concentrations that do not cause intrarenal crystal formation, may exacerbate renal injury in a model of AKI. 
In a prospective, randomized pilot study of 26 hyperuricemic patients undergoing CS, treatment with rasburicase resulted in decreased incidence of AKI ( $7.7 \%$ treatment group vs. $30.8 \%$ control group, $p=0.688$ ) [44]. Although not statistically significant, these results are consistent with a potential benefit of uric-acid-lowering therapy to reduce the risk of AKI. When analyzed by urine neutrophil gelatinase-associated lipocalin concentrations, the treatment group had less evidence of renal structural injury, especially in subjects with higher SUA levels and more severe renal dysfunction (baseline GFR $\leq 45 \mathrm{~mL} / \mathrm{min} / 1.73 \mathrm{~m}^{2}$ ) or heart failure (left ventricular ejection fraction $\leq 45 \%$ ).

In a study of 76 children with newly diagnosed advanced mature B cell non-Hodgkin lymphoma, lowering SUA with rasburicase was associated with a reduction in the incidence of AKI and TLS and increase in eGFR (average eGFR of $55 \mathrm{~mL} / \mathrm{min} / 1.73 \mathrm{~m}^{2}$ on day -1 up to an average of $136 \mathrm{~mL} / \mathrm{min} / 1.73 \mathrm{~m}^{2}$ on day 7 following treatment) [45]. These findings are better appreciated in the context that ischemic AKI involves the loss of renal autoregulation with enhanced levels of vasoconstrictors leading to hypoperfusion and ischemia/reperfusion injury.

In a prospective, randomized, controlled trial, lowering SUA with allopurinol in asymptomatic hyperuricemic patients with normal renal function was associated with improvement in endothelial dysfunction (flow-mediated dilatation in allopurinol group $7.74 \pm 0.93 \%$ vs. $7.76 \pm 0.86 \%$ control, $p<0.01$ ) and eGFR (allopurinol group $86.3 \pm 19.4 \mathrm{~mL} / \mathrm{min} / 1.73 \mathrm{~m}^{2}$ vs. control $84.3 \pm 16.7$ $\mathrm{mL} / \mathrm{min} / 1.73 \mathrm{~m}^{2}, p=0.04$ ) [46]. In 2 separate prospective, randomized, controlled trials of 159 and 500 patients, pretreatment with allopurinol and/or hydration prior to cardiac catheterizations/interventions was associated with significant decreases in SCreat levels $[47,48]$.

\section{Analogy}

The Effect of Similar Factors May be Considered

There is strong evidence for a causal relationship between uric acid and AKI in TLS via crystal-dependent mechanisms that involve mechanical tubular obstruction, tubular injury, and inflammatory pathways. Mesoamerican nephropathy involves repeated AKI from intermittent hyperuricemia and uricosuria, the effects of vasopressin, or the endogenous polyol fructokinase pathway [49]. AKI associated with SUA occurs without intratubular crystal deposition in CS and radiocontrast nephropathy. Some of the common mechanisms of SUAinduced diabetic nephropathy, hypertension, cardiovascular disease, chronic kidney disease, metabolic syndrome, and AKI include impaired endothelial function, stimulating endothelin-1, activating both the renal and intracellular renin angiotensin system, stimulating intracellular oxidative stress in the cytosol and mitochondria, stimulating smooth muscle cell proliferation and proinflammatory effects, and blocking endothelial nitric oxide release.

\section{Limitations, Conclusions, and Next Steps}

The difficulty with SUA is that it may accumulate from a fall in GFR, and hence has long been thought to be secondary. However, even preoperative studies show that SUA is a strong predictor for postoperative AKI independent of baseline renal function, suggesting that it can predict AKI even before AKI occurs, thereby making it less likely a secondary phenomenon.

\section{Summary}

In summary, studies have demonstrated that SUA is an independent predictor of AKI. Whether assessed by conventional or novel biomarkers, SUA has an inverse relationship with KeGFR and lowering SUA attenuates renal injury. The Bradford Hill criteria support the hypothesis for a causality of SUA in AKI. What is really needed now are double-blind, placebo-controlled trials to formally test this hypothesis.

\section{Disclosure Statement}

A.A.E., M.S., R.M., K.F.A., T.M.B., V.L., and B.D. do not have any conflicts of interest to disclose. R.J.J. is an inventor on patents related to lowering uric acid as a means to treat insulin resistance and diabetic nephropathy and has equity with XORT therapeutics, which is developing xanthine oxidase inhibitors for treatment of metabolic and kidney diseases.

The authors declare that the results presented in this paper have not been published previously in whole or part, except in the abstract format.

\section{Funding Sources}

This study received no grant support. 


\section{References}

1 Ames BN, Cathcart R, Schwiers E, Hochstein $P$. Uric acid provides an antioxidant defense in humans against oxidant- and radical-caused aging and cancer: a hypothesis. Proc Natl Acad Sci USA. 1981 Nov;78(11):6858-62.

2 Johnson RJ, Bakris GL, Borghi C, Chonchol MB, Feldman D, Lanaspa MA, et al. Hyperuricemia, Acute and Chronic Kidney Disease, Hypertension, and Cardiovascular Disease: Report of a Scientific Workshop Organized by the National Kidney Foundation. Am J Kidney Dis. 2018 Jun;71(6):851-65.

3 Hill AB. The environment and disease: association or causation? Proc R Soc Med. 1965 May;58:295-300.

4 Fedak KM, Bernal A, Capshaw ZA, Gross S. Applying the Bradford Hill criteria in the 21st century: how data integration has changed causal inference in molecular epidemiology. Emerg Themes Epidemiol. 2015 Sep;12(1):14.

5 Kjellstrand CM, Cambell DC 2nd, von Hartitzsch B, Buselmeier TJ. Hyperuricemic acute renal failure. Arch Intern Med. 1974 Mar; 133(3):349-59.

6 Ejaz AA, Mu W, Kang DH, Roncal C, Sautin YY, Henderson G, et al. Could uric acid have a role in acute renal failure? Clin J Am Soc Nephrol. 2007 Jan;2(1):16-21.

7 Ejaz AA, Beaver TM, Shimada M, Sood P, Lingegowda V, Schold JD, et al. Uric acid: a novel risk factor for acute kidney injury in high-risk cardiac surgery patients? Am J Nephrol. 2009;30(5):425-9.

8 Lapsia V, Johnson RJ, Dass B, Shimada M, Kambhampati G, Ejaz NI, et al. Elevated uric acid increases the risk for acute kidney injury. Am J Med. 2012 Mar;125(3):302.e9-17.

9 Ejaz AA, Kambhampati G, Ejaz NI, Dass B, Lapsia V, Arif AA, et al. Post-operative serum uric acid and acute kidney injury. J Nephrol. 2012 Jul-Aug;25(4):497-505.

10 Ejaz AA, Alquadan KF, Dass B, Shimada M, Kanbay M, Johnson RJ. Effects of serum uric acid on estimated GFR in CS patients: a pilot study. Am J Nephrol. 2015;42(6):402-9.

11 Lee EH, Choi JH, Joung KW, Kim JY, Baek SH, Ji SM, et al. Relationship between Serum Uric Acid Concentration and Acute Kidney Injury after Coronary Artery Bypass Surgery. J Korean Med Sci. 2015 Oct;30(10):1509-16.

12 Gaipov A, Solak Y, Turkmen K, Toker A, Baysal AN, Cicekler H, et al. Serum uric acid may predict development of progressive acute kidney injury after open heart surgery. Ren Fail. 2015 Feb;37(1):96-102.

13 Koratala A, Singhania G, Alquadan KF, Shimada M, Johnson RJ, Ejaz AA. Serum uric acid exhibits inverse relationship with estimated glomerular filtration rate. Nephron. 2016;134(4):231-7.

14 Ejaz AA, Pourafshar N, Mohandas R, Smallwood BA, Johnson RJ, Hsu JW. Uric acid and the prediction models of tumor lysis syndrome in AML. PLoS One. 2015 Mar; 10(3):e0119497.
15 Joung KW, Jo JY, Kim WJ, Choi DK, Chin JH, Lee $\mathrm{EH}$, et al. Association of preoperative uric acid and acute kidney injury following cardiovascular surgery. J Cardiothorac Vasc Anesth. 2014 Dec;28(6):1440-7.

16 Ben-Dov IZ, Kark JD. Serum uric acid is a GFR-independent long-term predictor of acute and chronic renal insufficiency: the Jerusalem Lipid Research Clinic cohort study. Nephrol Dial Transplant. 2011 Aug;26(8): 2558-66.

17 Otomo K, Horino T, Miki T, Kataoka H, Hatakeyama Y, Matsumoto T, et al. Serum uric acid level as a risk factor for acute kidney injury in hospitalized patients: a retrospective database analysis using the integrated medical information system at Kochi Medical School hospital. Clin Exp Nephrol. 2016 Apr;20(2): 235-43.

18 Nie S, Feng Z, Tang L, Wang X, He Y, Fang J, et al. Risk Factor Analysis for AKI Including Laboratory Indicators: a Nationwide Multicenter Study of Hospitalized Patients. Kidney Blood Press Res. 2017;42(5):761-73.

19 Greenberg KI, McAdams-DeMarco MA, Köttgen A, Appel LJ, Coresh J, Grams ME. Plasma Urate and Risk of a Hospital Stay with AKI: The Atherosclerosis Risk in Communities Study. Clin J Am Soc Nephrol. 2015 May; 10(5):776-83

20 Joung KW, Choi SS, Kong YG, Yu J, Lim J, Hwang $\mathrm{JH}$, et al. Incidence and risk factors of acute kidney injury after radical cystectomy: importance of preoperative serum uric acid level. Int J Med Sci. 2015 Jul;12(7):599-604.

$21 \mathrm{Yu}$ J, Park HK, Kwon HJ, Lee J, Hwang $\mathrm{JH}$, Kim HY, et al. Risk factors for acute kidney injury after percutaneous nephrolithotomy: implications of intraoperative hypotension. Medicine (Baltimore). 2018 Jul; 97(30):e11580.

22 Kanbay M, Solak Y, Afsar B, Nistor I, Aslan G, Çağlayan OH, et al. Serum Uric Acid and Risk for Acute Kidney Injury Following Contrast. Angiology. 2017 Feb;68(2):132-44.

23 Liang J, Zhang P, Hu X, Zhi L. Elevated serum uric acid after injury correlates with the early acute kidney in severe burns. Burns. 2015 Dec;41(8):1724-31.

24 Kanbay M, Jensen T, Solak Y, Le M, RoncalJimenez C, Rivard C, et al. Uric acid in metabolic syndrome: from an innocent bystander to a central player. Eur J Intern Med. 2016 Apr;29:3-8.

25 Cicerchi C, Li N, Kratzer J, Garcia G, RoncalJimenez CA, Tanabe K, et al. Uric acid-dependent inhibition of AMP kinase induces hepatic glucose production in diabetes and starvation: evolutionary implications of the uricase loss in hominids. FASEB J. 2014 Aug;28(8): 3339-50.

26 Sautin YY, Nakagawa T, Zharikov S, Johnson RJ. Adverse effects of the classic antioxidant uric acid in adipocytes: NADPH oxidase-mediated oxidative/nitrosative stress. Am J
Physiol Cell Physiol. 2007 Aug;293(2):C58496.

27 Roncal-Jimenez CA. Lanaspa MA, Rivard CJ, Nakagawa T, Sanchez-Lozada LG, Jalal D, et al. Sucrose induces fatty liver and pancreatic inflammation in male breeder rats independent of excess energy intake. Metabolism. 2011 Sep;60(9):1259-70.

28 Lanaspa MA. Sanchez-Lozada LG, Choi YJ, Cicerchi C, Kanbay M, Roncal-Jimenez CA, et al. Uric acid induces hepatic steatosis by generation of mitochondrial oxidative stress: potential role in fructose-dependent and -independent fatty liver. J Biol Chem. 2012 Nov; 287(48):40732-44.

29 Kaufeld T, Foerster KA, Schilling T, Kielstein JT, Kaufeld J, Shrestha M, et al. Preoperative serum uric acid predicts incident acute kidney injury following cardiac surgery. BMC Nephrol. 2018 Jul;19(1):161.

30 Liu Y, Tan N, Chen J, Zhou Y, Chen L, Chen $\mathrm{S}$, et al. The relationship between hyperuricemia and the risk of contrast-induced acute kidney injury after percutaneous coronary intervention in patients with relatively normal serum creatinine. Clinics (São Paulo). 2013 Jan;68(1):19-25.

31 Spencer HW, Yarger WE, Robinson RR. Alterations of renal function during dietary-induced hyperuricemia in the rat. Kidney Int. 1976 Jun;9(6):489-500.

32 Mulay SR, Shi C, Ma X, Anders HJ. Novel Insights into Crystal-Induced Kidney Injury. Kidney Dis (Basel). 2018 Jun;4(2):49-57.

33 Sánchez-Lozada LG, Tapia E, Santamaría J, Avila-Casado C, Soto V, Nepomuceno T, et al. Mild hyperuricemia induces vasoconstriction and maintains glomerular hypertension in normal and remnant kidney rats. Kidney Int. 2005 Jan;67(1):237-47.

34 Kang DH, Park SK, Lee IK, Johnson RJ. Uric acid-induced C-reactive protein expression: implication on cell proliferation and nitric oxide production of human vascular cells. J Am Soc Nephrol. 2005 Dec;16(12):3553-62.

35 Kang DH, Han L, Ouyang X, Kahn AM, Kanellis J, Li P, et al. Uric acid causes vascular smooth muscle cell proliferation by entering cells via a functional urate transporter. Am J Nephrol. 2005 Sep-Oct;25(5):425-33.

36 Kanellis J, Watanabe S, Li JH, Kang DH, Li P, Nakagawa T, et al. Uric acid stimulates monocyte chemoattractant protein-1 production in vascular smooth muscle cells via mitogen-activated protein kinase and cyclooxygenase- 2 . Hypertension. 2003 Jun;41(6):1287-93.

37 Khosla UM, Zharikov S, Finch JL, Nakagawa $\mathrm{T}$, Roncal C, Mu W, et al. Hyperuricemia induces endothelial dysfunction. Kidney Int. 2005 May;67(5):1739-42.

38 Nakagawa T, Hu H, Zharikov S, Tuttle KR, Short RA, Glushakova O, et al. A causal role for uric acid in fructose-induced metabolic syndrome. Am J Physiol Renal Physiol. 2006 Mar;290(3):F625-31. 
39 Kanbay M, Siriopol D, Nistor I, Elcioglu OC, Telci O, Takir M, et al. Effects of allopurinol on endothelial dysfunction: a meta-analysis. Am J Nephrol. 2014;39(4):348-56.

40 Agarwal A, Dong Z, Harris R, Murray P, Parikh SM, Rosner MH, et al.; Acute Dialysis Quality Initiative XIII Working Group. Cellular and Molecular Mechanisms of AKI. J Am Soc Nephrol. 2016 May;27(5):1288-99.

41 Shimada M, Johnson RJ, May WS Jr, Lingegowda V, Sood P, Nakagawa T, et al. A novel role for uric acid in acute kidney injury associated with tumour lysis syndrome. Nephrol Dial Transplant. 2009 Oct;24(10):2960-4.

42 Shimada M, Dass B, Ejaz AA. Paradigm shift in the role of uric acid in acute kidney injury. Semin Nephrol. 2011 Sep;31(5):453-8.

43 Roncal CA, Mu W, Croker B, Reungjui S, Ouyang X, Tabah-Fisch I, et al. Effect of elevated serum uric acid on cisplatin-induced acute renal failure. Am J Physiol Renal Physiol. 2007 Jan;292(1):F116-22.
44 Ejaz AA, Dass B, Lingegowda V, Shimada M, Beaver TM, Ejaz NI, et al. Effect of uric acid lowering therapy on the prevention of acute kidney injury in cardiovascular surgery. Int Urol Nephrol. 2013 Apr;45(2):44958.

45 Galardy PJ, Hochberg J, Perkins SL, Harrison L, Goldman S, Cairo MS. Rasburicase in the prevention of laboratory/clinical tumour lysis syndrome in children with advanced mature B-NHL: a Children's Oncology Group Report. Br J Haematol. 2013 Nov;163(3): 365-72.

46 Kanbay M, Huddam B, Azak A, Solak Y, Kadioglu GK, Kirbas I, et al. A randomized study of allopurinol on endothelial function and estimated glomular filtration rate in asymptomatic hyperuricemic subjects with normal renal function. Clin J Am Soc Nephrol. 2011 Aug; 6(8):1887-94.

47 Erol T, Tekin A, Katırcıbaşı MT, Sezgin N, Bilgi M, Tekin G, et al. Efficacy of allopurinol pretreatment for prevention of contrast-induced nephropathy: a randomized controlled trial. Int J Cardiol. 2013 Aug; 167(4): 1396-9.

48 Kumar A, Bhawani G, Kumari N, Murthy KS, Lalwani V, Raju CN. Comparative study of renal protective effects of allopurinol and $\mathrm{N}$ acetyl-cysteine on contrast induced nephropathy in patients undergoing cardiac catheterization. J Clin Diagn Res. 2014 Dec; 8(12):HC03-07.

49 Roncal Jimenez CA, Ishimoto T, Lanaspa MA, Rivard CJ, Nakagawa T, Ejaz AA, et al. Fructokinase activity mediates dehydrationinduced renal injury. Kidney Int. 2014 Aug; 86(2):294-302.

50 Kim JH, Gil HW, Yang JO, Lee EY, Hong SY. Serum uric acid level as a marker for mortality and acute kidney injury in patients with acute paraquat intoxication. Nephrol Dial Transplant. 2011 Jun;26(6): 1846-52. 